\title{
THE FRACTIONAL HILBERT TRANSFORM AND DUAL-TREE GABOR-LIKE WAVELET ANALYSIS
}

\author{
Kunal Narayan Chaudhury and Michael Unser \\ Biomedical Imaging Group, \\ Ecole Polytechnique Fédérale de Lausanne (EPFL), CH-1015, Switzerland.
}

\begin{abstract}
We provide an amplitude-phase representation of the dual-tree complex wavelet transform by extending the fixed quadrature relationship of the dual-tree wavelets to arbitrary phase-shifts using the fractional Hilbert transform (fHT). The fHT is a generalization of the Hilbert transform that extends the quadrature phase-shift action of the latter to arbitrary phase-shifts-a real shift parameter controls this phase-shift action.

Next, based on the proposed representation and the observation that the fHT operator maps well-localized B-spline wavelets (that resemble Gaussian-windowed sinusoids) into B-spline wavelets of the same order but different shift, we relate the corresponding dual-tree scheme to the paradigm of multiresolution windowed Fourier analysis.
\end{abstract}

Index Terms - Fractional Hilbert Transform, DualTree Complex Wavelet Transform (DT-CWT), B-spline Wavelet, Gabor Function.

\section{INTRODUCTION}

$\mathrm{I}^{\mathrm{N}}$ this paper, we study the family of fractional Hilbert transforms that generalize the Hilbert transform operator [1] by extending the phase-shift action $\cos \left(\omega_{0} x\right) \mapsto$ $\cos \left(\omega_{0} x+\theta\right)$ of the latter from $\theta=-\pi / 2$ to any arbitrary $\theta$ in $[0,2 \pi]$. More crucially, they exhibit simultaneous translation and dilation invariance, which allows us to integrate them easily into the multiresolution framework of wavelets [cf. §2].

In particular, using the fHT as the generic phase-shift operator, we propose a novel amplitude-phase interpretation of the dual-tree complex wavelet transform. The DT-CWT is a recent enhancement to the discrete wavelet transform that has gained increasing popularity as a signal processing tool. This was originally introduced by Kingsbury [2] to overcome the shift-variance problem of the conventional discrete wavelet transform (DWT). The

Correspondence: \{kunal.chaudhury,michael.unser\}@epfl.ch. This work was partly supported by the Swiss National Science Foundation under grant 200020-109415. crucial observation that the dual-tree wavelets form an approximate Hilbert transform (HT) pair was made by Selesnick [3]; this consequently reduced the design of different flavors of dual-tree wavelets to the construction of new HT pairs of wavelets $[3,4,5]$. We refer the reader to the excellent tutorial [6] on the design and application of the dual-tree transform.

The main goal of this paper is to formally link the dual-tree paradigm with the amplitude-phase formalism of Fourier analysis. Specifically, by identifying the dualtree wavelet pair $(\psi, \mathscr{H} \psi)$ with the complex wavelet

$$
\Psi(x)=\psi(x)+j \mathscr{H} \psi(x)
$$

and, based on the polar representation $c_{n}=\left|c_{n}\right| e^{j \phi_{n}}$ of the complex dual-tree coefficients $c_{n}=\left\langle f, \Psi_{n}\right\rangle$, we establish the following representation

$$
f(x)=\sum\left|c_{n}\right| \psi_{n, \tau_{n}}(x),
$$

resembling the Fourier decomposition

$$
g(x)=\sum\left|c_{n}\right| \cos \left(n \omega_{0} x+\phi_{n}\right)
$$

of a periodic signal $g(x)$ [cf. §3]. The significance of the fHT is that the wavelet $\psi_{n, \tau_{n}}(x)$ in (1) is derived from the reference wavelet $\psi_{n}(x)$ via the transformation

$$
\psi_{n, \tau_{n}}(x)=\mathcal{H}_{\tau_{n}} \psi_{n}(x)
$$

where $\mathcal{H}_{\tau_{\mathrm{n}}}$ is the fHT operator corresponding to the shift $\tau_{n}=\phi_{n} / \pi$. Finally, an explicit understanding of the action of the fHT is arrived at for the particular family of dual-tree transform involving HT pairs of B-spline wavelets [5], that asymptotically converge to Gabor-like functions [7].

\section{THE FRACTIONAL HILBERT TRANSFORM}

The fHT forms the cornerstone of the our subsequent discussion and we begin with a detailed study of its characteristics. There exits several definitions of the fHT in the signal processing and optics literature $[8,9,10,11]$; 
however, for reasons that will become obvious in the sequel, we propose an operator-based formulation involving the interpolation of the 'quadrature' identity operator $\mathcal{J}$ and HT operator $\mathscr{H}$. In particular, we define the fHT operator $\mathcal{H}_{\tau}$, corresponding to the shift parameter $\tau \in \mathbb{R}$, as

$$
\mathcal{H}_{\tau}=\cos (\pi \tau) \mathcal{J}-\sin (\pi \tau) \mathscr{H}
$$

The above definition is equivalent to the formulations used in $[10,11]$, but differs from the ones in $[8,9]$ up to a complex chirp. The advantage of using the above formulation will be evident in the sequel.

\subsection{Characterization of the fHT}

The fHT operator inherits most of the characteristic features of the constituent identity and HT operators, which happen to be two special members of the fHT family; specifically, $\mathcal{J}=\mathcal{H}_{0}$ and $\mathscr{H}=\mathcal{H}_{-1 / 2}$. Based on (3) and the properties of the identity and HT operator, the following mathematical properties of the fHT can be readily derived:

(P1) $\mathcal{H}_{\tau}$ is linear and translation invariant; that is, it acts as a convolution operator.

(P2) It commutes with dilations, so that $\left(\mathcal{H}_{\tau} f\right)(x / a)=$ $\mathcal{H}_{\tau}\{f(\cdot / a)\}(x)$, for all $a>0$.

(P3) It is unitary; i.e, $\left\|\mathcal{H}_{\tau} f\right\|=\|f\|$, for all $f \in L^{2}(\mathbb{R})$.

(P4) It acts as a phase-shift operator on pure sinusoids; specifically, $\mathcal{H}_{\tau} \cos \left(\omega_{0} x\right)=\cos \left(\omega_{0} x+\pi \tau\right)$.

(P5) It satisfies the composition law $\mathcal{H}_{\tau_{1}} \mathcal{H}_{\tau_{2}}=\mathcal{H}_{\tau_{1}+\tau_{2}}$. In particular, $\mathcal{H}_{\tau}^{\mathrm{m}}=\mathcal{H}_{\mathrm{m} \tau}$, for any integer $\mathrm{m}$.

The significance of (P3) is that the inverse fHT operator is well-defined and is given by its own adjoint; moreover, the property $\mathcal{H}_{\tau} \mathcal{H}_{-\tau}=\mathcal{H}_{0}=\mathcal{J}$, yields the relation $\mathcal{H}_{\tau}^{-1}=\mathcal{H}_{\tau}^{*}=\mathcal{H}_{-\tau}$. In this context, (P5) must then be interpreted for any negative integer $m=-n, n>0$, as $\left(\mathcal{H}_{\tau}^{-1}\right)^{n}=\mathcal{H}_{m \tau}$. These composition properties are succinctly captured by the following statement:

Proposition 2.1 The family of linear operators $\left\{\mathcal{H}_{\tau}\right\}_{\tau \in \mathbb{R}}$ form a commutative group on $\mathrm{L}^{2}(\mathbb{R})$.

Next, we introduce the following fractional extension of the Bedrosian identity [12].

Theorem 2.2 (Generalized Bedrosian Identity) Let $f(x)$ and $\mathrm{g}(\mathrm{x})$ be real-valued functions such that the support of $\hat{f}(\omega)$ is restricted to $(-\Omega, \Omega)$, and that $\hat{g}(\omega)$ vanishes for $|\omega|<\Omega$, for some arbitrary frequency $\Omega$. Then the fHT of the product function $f(x) g(x)$ can be expressed as

$$
\mathcal{H}_{\tau}\{f(x) g(x)\}=f(x) \mathcal{H}_{\tau} g(x) .
$$

Proof. This follows as a consequence of the Bedrosian identity $\mathscr{H}\{\mathrm{f}(\mathrm{x}) \mathrm{g}(\mathrm{x})\}=\mathrm{f}(\mathrm{x}) \mathscr{H} \mathrm{g}(\mathrm{x})$, and the definition of the fHT. Indeed, we have

$$
\begin{aligned}
\mathcal{H}_{\tau}\{\mathrm{f}(\mathrm{x}) \mathrm{g}(\mathrm{x})\} & =\cos (\pi \tau) \mathrm{f}(\mathrm{x}) \mathrm{g}(\mathrm{x})-\sin (\pi \tau) \mathscr{H}\{\mathrm{f}(\mathrm{x}) \mathrm{g}(\mathrm{x})\} \\
& =\cos (\pi \tau) \mathrm{f}(\mathrm{x}) \mathrm{g}(\mathrm{x})-\sin (\pi \tau) \mathrm{f}(\mathrm{x}) \mathscr{H} \mathrm{g}(\mathrm{x}) \\
& =\mathrm{f}(\mathrm{x}) \mathcal{H}_{\tau} \mathrm{g}(\mathrm{x}) .
\end{aligned}
$$

An immediate consequence of theorem (2.2) and property (P4) is that for a bandlimited signal $\varphi(x)$ with frequency support restricted to $\left(-\omega_{0}, \omega_{0}\right)$, one has the following modulation property

$$
\mathcal{H}_{\tau}\left\{\varphi(x) \cos \left(\omega_{0} x\right)\right\}=\varphi(x) \cos \left(\omega_{0} x+\pi \tau\right) .
$$

It turns out that, similar to the Hilbert transform, the fHT fits in well into the wavelet formalism. Following the fact that the HT maps a wavelet into a wavelet, it can be easily verified that the fHT of a wavelet basis is a valid wavelet basis as well [5]. In particular, the implications of the shift and dilation invariance is that the fHT of shifted-dilated wavelets are wavelets shifted and dilated by the same amount; these invariance properties facilitate the incorporation of the fHT into the wavelet framework.

\section{AMPLITUDE-PHASE REPRESENTATION OF THE DUAL-TREE TRANSFORM}

Next, using the fHT as the phase-shift operator $f(x) \mapsto$ $\mathcal{H}_{\tau} f(x)$, we provide a amplitude-phase interpretation of the DT-CWT similar to the Fourier analysis of periodic signals. The Fourier decomposition of a signal $f(x)$ in $\mathrm{L}^{2}([0, \mathrm{~T}])$, in terms of pure sinusoids, is given by

$$
\begin{aligned}
f(x)=a_{0} & +a_{1} \cos \left(\omega_{0} x\right)+a_{2} \cos \left(2 \omega_{0} x\right)+\cdots \\
& +b_{1} \sin \left(\omega_{0} x\right)+b_{2} \sin \left(2 \omega_{0} x\right)+\cdots
\end{aligned}
$$

where $\omega_{0}\left(\omega_{0} T=2 \pi\right)$ is the fundamental frequency, and where $a_{0}, a_{1}, a_{2}, \ldots, b_{1}, b_{2}, \ldots$ are the Fourier coefficients corresponding to the different harmonics. Note that this admits the following alternative representation

$$
\begin{aligned}
f(x) & =\sum\left|c_{n}\right| \cos \left(n \omega_{0} x+\phi_{n}\right) \\
& =\sum\left|c_{n}\right| \mathcal{H}_{\tau_{n}}\left\{\cos \left(n \omega_{0} x\right)\right\},
\end{aligned}
$$

based on the fHT, with $c_{n}=a_{n}-j b_{n}=\left|c_{n}\right| e^{j \phi_{n}}$ being the polar representation of the complex Fourier coefficient $c_{n}=\left\langle f(x), e^{j n \omega_{0} x}\right\rangle$, and where $\tau_{n}=\phi_{n} / \pi$. The role of the $\mathrm{fHT}$ in (7) is to provide the correct phaseshifts to the reference functions $\left\{\cos \left(n \omega_{0} x\right)\right\}$.

Our present contribution is to transpose the amplitudephase representation to the DT-CWT, using quadrature pairs of wavelet instead of the conjugate sinusoid-pairs: 
$\left\{\cos \left(n \omega_{0} x\right)\right\}$ and $\left\{\sin \left(n \omega_{0} x\right)\right\}$. In particular, the DT-CWT decomposes a signal $f(x)$ in $L^{2}(\mathbb{R})$ using two parallel wavelet bases $\left\{\psi_{n}\right\}_{n \in \mathbb{N}}$ and $\left\{\mathscr{H} \psi_{n}\right\}_{n \in \mathbb{N}}$-derived via dilations and translations of the quadrature wavelets $\psi(x)$ and $\mathscr{H} \psi(x)$-yielding the following equivalent expansions

$$
f(x)= \begin{cases}\sum a_{n} \psi_{n}(x), & (\text { Branch 1) } \\ \sum b_{n} \mathscr{H} \psi_{n}(x), & (\text { Branch 2) }\end{cases}
$$

The wavelet coefficients of the respective branches, $a_{n}=\left\langle f, \widetilde{\psi}_{n}\right\rangle$ and $b_{n}=\left\langle f, \mathscr{H} \widetilde{\psi}_{n}\right\rangle$, are obtained via the projection of the signal onto the corresponding analysis (dual) wavelet bases $\left\{\widetilde{\psi}_{n}\right\}_{n \in \mathbb{N}}$ and $\left\{\mathscr{H} \widetilde{\psi}_{n}\right\}_{n \in \mathbb{N}}$, respectively [5].

Based on the polar representation $\left|c_{n}\right| e^{j \phi_{n}}$ of the complex wavelet coefficients $c_{n}=\left(a_{n}-j b_{n}\right) / 2$, and by combining the expansions in (8), we arrive at the following amplitude-phase representation

$$
\begin{aligned}
f(x) & =\frac{1}{2} \sum\left(a_{n} \psi_{n}(x)+b_{n} \mathscr{H} \psi_{n}(x)\right) \\
& =\sum\left|c_{n}\right|\left(\cos \phi_{n} \psi_{n}(x)-\sin \phi_{n} \mathscr{H} \psi_{n}(x)\right) \\
& =\sum\left|c_{n}\right| \psi_{n, \tau_{n}}(x),
\end{aligned}
$$

where the shift parameters are specified as $\tau_{n}=\phi_{n} / \pi$.

The key identification is that the transformed wavelets $\psi_{n, \tau_{n}}(x)=\mathcal{H}_{\tau_{n}} \psi_{n}(x)$ in (9) - the 'phase-shifted' versions of the primary wavelets $\left\{\psi_{n}\right\}$-play a role similar to the 'optimally' displaced sinusoids $\left\{\cos \left(n \omega_{0} x+\phi_{n}\right)\right\}$ in (7). Based on this representation, one can then interpret the dual-tree transform $f(x) \mapsto\left\{\left(\left|c_{n}\right|, \phi_{n}\right)\right\}_{n \in \mathbb{N}}$ as some form of multiresolution amplitude-phase analysis of the signal $f(x)$. Moreover, as discussed next, the wavelets $\psi_{n, \tau_{n}}(x)$ in (9) can be explicitly characterized for particular flavors of spline wavelets.

\section{WINDOWED FOURIER-LIKE MULTIRESOLUTION ANALYSIS}

The theoretical construction of exact HT pairs of biorthogonal wavelets and Gabor-like wavelets using B-spline wavelets, in particular, was recently discussed in [5]. It turns out that the fHT of a B-spline wavelet can be well-characterized as well.

The semi-orthogonal B-spline wavelet is of special interest as it is better localized in space than its orthogonal counterpart; it exhibits remarkable joint time-frequency localization properties [7]. Starting from the fractional B-spline scaling function $\beta_{\tau}^{\alpha}(x)$, the transfer function of the wavelet filter that generates the 'shortest' B-spline wavelet $\psi_{\tau}^{\alpha}(x)$ (orthogonal to the shifts of $\beta_{\tau}^{\alpha}(x)$ but not to its own shifts) is then specified as

$$
G\left(e^{j \omega}\right)=e^{j \omega} A\left(-e^{j \omega}\right) H\left(-e^{-j \omega}\right),
$$

where $\mathrm{H}\left(\mathrm{e}^{j \omega}\right)$ and $A\left(\mathrm{e}^{j \omega}\right)$ are the refinement filter and autocorrelation filter of $\beta_{\tau}^{\alpha}(x)$, respectively $[7,13]$. The associated biorthogonal system comprises of the (unique) dual-spline scaling function $\AA_{\tau}^{\alpha}(x)$ and the dual wavelet $\widetilde{\psi}_{\tau}^{\alpha}(x)$, satisfying the biorthogonality condition $\left\langle\psi_{\tau}^{\alpha}, \widetilde{\psi}_{\tau}^{\alpha}(\cdot-n)\right\rangle=\delta[n]$. It turns out that the $(\alpha, \tau)$-family of fractional B-spline wavelets [13] and their duals are closed with respect to the fHT operator.

Proposition 4.1 The fHT of a B-spline wavelet is another $B$-spline wavelet of same order but different shift:

$$
\mathcal{H}_{\bar{\tau}} \psi_{\tau}^{\alpha}(x)=\psi_{\tau-\bar{\tau}}^{\alpha}(x) .
$$

Moreover, the dual wavelet satisfies an identical relation.

Without loss of generality, we shall henceforth restrict our discussion to the wavelet $\psi^{\alpha}(x)=\psi_{0}^{\alpha}(x)$ associated with the symmetric B-spline $\beta_{0}^{\alpha}(x)$. It is well known that the B-spline wavelet asymptotically converges $^{1}$ to a modulated Gaussian [7]; in particular

$$
\psi^{\alpha}(x) \sim \varphi^{\alpha}(x) \cos \left(\omega_{0} x+\xi_{0}\right),
$$

as $\alpha \rightarrow+\infty$, where $\varphi^{\alpha}(x)$ denotes a Gaussian window that is completely characterized by the degree of the Bspline, whereas, the frequency and phase parameters $\omega_{0}$ and $\xi_{0}$ are independent of $\alpha$.

Based on the modulation property (5), one then has the following asymptotic relation

$$
\mathcal{H}_{\tau} \psi^{\alpha}(x) \sim \varphi^{\alpha}(x) \cos \left(\omega_{0} x+\xi_{0}+\pi \tau\right)
$$

as $\alpha \rightarrow+\infty$; that is, the fHT acts only on the the phase of the modulating sinusoid, preserving the Gaussianlike envelope. It is worth noting that the asymptotic form in (13) is in fact the real part of the Gabor function $\varphi^{\alpha}(x) \exp \left(j\left(\omega_{0} x+\xi_{0}+\pi \tau\right)\right)$. Figure 1 provides an illustrations of these results. It shows pairs $\left(\mathcal{H}_{\tau} \psi^{8}(x), \mathcal{H}_{\tau+\frac{1}{2}} \psi^{8}(x)\right)$ of quadrature B-spline wavelets, corresponding to the different shifts of the reference wavelet; the Gaussian-like envelope $\mid \mathcal{H}_{\tau} \psi^{8}(x)+$ $j \mathcal{H}_{\tau+\frac{1}{2}} \psi^{8}(x) \mid$ is also depicted for each $\tau$.

Now, for the dual-tree analysis involving the B-spline wavelet and its HT pair, (9) leads to the following explicit form

$$
f(x)=\sum\left|c_{n}\right| \mathcal{H}_{\tau_{n}} \psi_{n}^{\alpha}(x)=\sum\left|c_{n}\right| \psi_{n,-\tau_{n}}^{\alpha}(x),
$$

where $\psi_{n,-\tau_{n}}^{\alpha}(x)$ is also a B-spline wavelet, 'shifted' from its reference $\psi_{n}^{\alpha}(x)$ by $-\tau_{n}$; indeed, in view of the asymptotic form (13), the action of the fHT translates into a phase-shift of the modulating sinusoid (for large orders). In this light, one can then interpret (14) as a form of multiresolution windowed Fourier-like representation of the signal $f(x)$.

\footnotetext{
${ }^{1}$ The asymptotic equality notation $f_{\alpha}(x) \sim g_{\alpha}(x)$ signifies that $\mathrm{f}_{\alpha}(\mathrm{x}) / \mathrm{g}_{\alpha}(\mathrm{x}) \rightarrow 1$ as $\alpha \rightarrow \infty$, for all $\mathrm{x}$.
} 


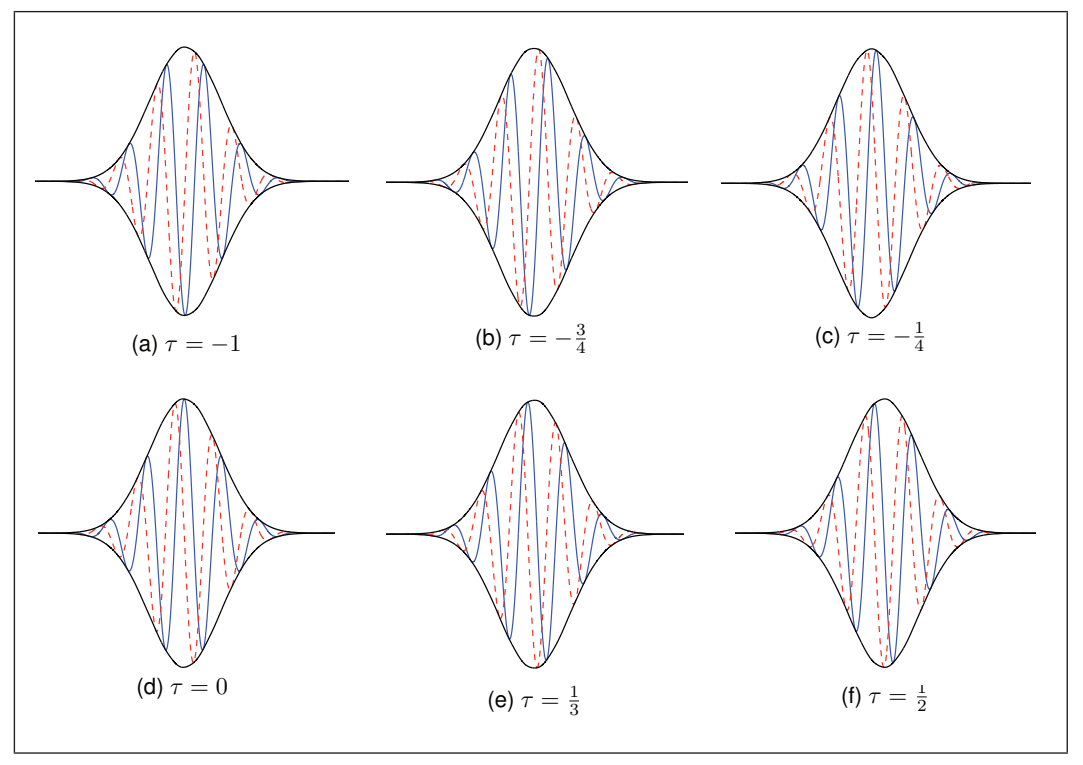

Fig. 1. Quadrature Pairs of B-spline Wavelets; Blue (solid line): $\mathcal{H}_{\tau} \psi^{8}(\mathrm{x})$; Red (broken line): $\mathcal{H}_{\tau+\frac{1}{2}} \psi^{8}(\mathrm{x})$; and Black (solid line): Common localization window given by $\left|\mathcal{H}_{\tau} \psi^{8}(x)+j \mathcal{H}_{\tau+\frac{1}{2}} \psi^{8}(x)\right|$.

\section{CONCLUSION}

An amplitude-phase representation of the DT-CWT was proposed using the fractional Hilbert transform. An explicit characterization of this was then provided in terms of the localized B-spline wavelets. These could be of potential interest in signal processing applications involving the dual-tree transform, particularly signal denoising, where a model linking the reconstructed signal to the processed complex wavelet coefficients is desirable.

\section{REFERENCES}

[1] R. Bracewell, The Fourier Transform and Its Applications, McGraw-Hill, 1986.

[2] N.G. Kingsbury, "Complex Wavelets for Shift Invariant Analysis and Filtering of Signals," Journal of Applied and Computational Harmonic Analysis, vol. 10, no. 3, pp. 234-253, May 2001.

[3] I.W. Selesnick, "Hilbert Transform Pairs of Wavelet Bases," IEEE Sig. Proc. Letters, vol. 8, no. 6, pp. 170-173, 2001.

[4] R. Yu and H. Ozkaramanli, "Hilbert Transform Pairs of Biorthogonal Wavelet Bases," IEEE Trans. on SP, vol. 54, pp. 2119-2125, June 2006.

[5] K.N. Chaudhury and M. Unser, "Construction of Hilbert Transform Pairs of Wavelet Bases and Optimal Time-Frequency Localization," Proceedings: ICASSP, pp. 3277-3280, April, 2008.
[6] I. W. Selesnick, R. G. Baraniuk, and N. C. Kingsbury, "The Dual-tree Complex Wavelet Transform," IEEE Sig. Proc. Magazine, vol. 22, no. 6, pp. 123151, November 2005.

[7] M. Unser, A. Aldroubi, and M. Eden, "On the Asymptotic Convergence of B-Spline Wavelets to Gabor Functions," IEEE Trans. on Info. Theory, vol. 38, no. 2, pp. 864-872, March 1992.

[8] R. Tao, X. M. Li, and Y. Wang, "Generalization of the Fractional Hilbert Transform," IEEE Sig. Proc. Letters, vol. 15, pp. 365-368, 2008.

[9] A.I. Zayed, "Hilbert Transform Associated with the Fractional Fourier Transform," IEEE Sig. Proc. Letters, vol. 5, pp. 206-208, 1998.

[10] J. A. Davis, D. E. McNamara, and D. M. Cottrell, "Analysis of the Fractional Hilbert Transform," Applied Optics, vol. 37, pp. 6911-6913, 1998.

[11] A. W. Lohmann, D. Mendlovic, and Z. Zalevsky, "Fractional Hilbert Transform," Optics Letters, vol. 21, pp. 281-283, 1996.

[12] E. Bedrosian, "A Product Theorem for Hilbert Transforms," Proc. IEEE, vol. 51, pp. 868-869, 1963.

[13] T. Blu and M. Unser, "A Complete Family of Scaling Functions: The $(\alpha, \tau)$-Fractional Splines," Proceedings: ICASSP, vol. 4, pp. 421-424, April 6-10 2003. 